\title{
An inequality for contact CR-warped product submanifolds of nearly cosymplectic manifolds
}

\author{
Siraj Uddin ${ }^{* *}$ and Khalid Ali Khan ${ }^{2}$
}

"Correspondence:

siraj.ch@gmail.com

${ }^{1}$ Institute of Mathematical Sciences,

Faculty of Science, University of

Malaya, Kuala Lumpur, 50603,

Malaysia

Full list of author information is

available at the end of the article

\begin{abstract}
Recently, Chen (Monatshefte Math. 133:177-195, 2001) established general sharp inequalities for CR-warped products in a Kaehler manifold. Afterward, Mihai obtained (Geom. Dedic. 109:165-173, 2004) the same inequalities for contact CR-warped product submanifolds of Sasakian space forms and derived some applications. In this paper, we obtain an inequality for the length of the second fundamental form of the warped product submanifold of a nearly cosymplectic manifold in terms of the warping function. The equality case is also discussed.
\end{abstract}

MSC: $53 C 40 ; 53 C 42 ; 53 B 25$

Keywords: warped product; contact CR-submanifold; contact CR-warped product; nearly cosymplectic manifold

\section{Introduction}

An almost contact metric structure $(\phi, \xi, \eta, g)$ satisfying $\left(\bar{\nabla}_{X} \phi\right) X=0$ is called a nearly cosymplectic structure. If we consider $S^{5}$ as a totally geodesic hypersurface of $S^{6}$, then it is known that $S^{5}$ has a non-cosymplectic nearly cosymplectic structure. Almost contact manifolds with Killing structure tensors were defined in [1] as nearly cosymplectic manifolds, and it was shown that the normal nearly cosymplectic manifolds are cosymplectic (see also [2]). Later on, Blair and Showers [3] studied nearly cosymplectic structure $(\phi, \xi, \eta, g)$ on a manifold $\bar{M}$ with $\eta$ closed from the topological viewpoint.

On the other hand, Chen [4] has introduced the notion of CR-warped product submanifolds in a Kaehler manifold. He has established a sharp relationship between the squared norm of the second fundamental form and the warping function. Later on, Mihai [5] studied contact CR-warped products and obtained the same inequality for contact CRwarped product submanifolds isometrically immersed in Sasakian space forms. Motivated by the studies of these authors, many articles dealing with the existence or non-existence of warped products in different settings have appeared; one of them is [3]. In this paper, we obtain an inequality for the length of the second fundamental form in terms of the warping function for contact CR-warped product submanifolds in a more general setting, i.e., nearly cosymplectic manifold. 


\section{Preliminaries}

A $(2 m+1)$-dimensional smooth manifold $\bar{M}$ is said to have an almost contact structure if on $\bar{M}$ there exist a tensor field $\phi$ of type $(1,1)$, a vector field $\xi$ and a 1-form $\eta$ satisfying [6]

$$
\phi^{2}=-I+\eta \otimes \xi, \quad \phi \xi=0, \quad \eta \circ \phi=0, \quad \eta(\xi)=1 .
$$

There always exists a Riemannian metric $g$ on $\bar{M}$ satisfying the following compatibility condition:

$$
\eta(X)=g(X, \xi), \quad g(\phi X, \phi Y)=g(X, Y)-\eta(X) \eta(Y),
$$

where $X$ and $Y$ are vector fields on $\bar{M}[6]$.

An almost contact structure $(\phi, \xi, \eta)$ is said to be normal if the almost complex structure $J$ on the product manifold $\bar{M} \times \mathbb{R}$ given by

$$
J\left(X, f \frac{d}{d t}\right)=\left(\phi X-f \xi, \eta(X) \frac{d}{d t}\right)
$$

where $f$ is a smooth function on $\bar{M} \times \mathbb{R}$, has no torsion, i.e., $J$ is integrable, the condition for normality in terms of $\phi, \xi$ and $\eta$ is $[\phi, \phi]+2 d \eta \otimes \xi=0$ on $\bar{M}$, where $[\phi, \phi]$ is the Nijenhuis tensor of $\phi$. Finally, the fundamental 2-form $\Phi$ is defined by $\Phi(X, Y)=g(X, \phi Y)$.

An almost contact metric structure $(\phi, \xi, \eta, g)$ is said to be cosymplectic if it is normal and both $\Phi$ and $\eta$ are closed [6]. The structure is said to be nearly cosymplectic if $\phi$ is Killing, i.e., if

$$
\left(\bar{\nabla}_{X} \phi\right) Y+\left(\bar{\nabla}_{Y} \phi\right) X=0
$$

for any $X, Y$ tangent to $\bar{M}$, where $\bar{\nabla}$ is the Riemannian connection of the metric $g$ on $\bar{M}$. Equation (2.3) is equivalent to $\left(\bar{\nabla}_{X} \phi\right) X=0$ for each vector field $X$ tangent to $\bar{M}$. The structure is said to be closely cosymplectic if $\phi$ is Killing and $\eta$ is closed. It is well known that an almost contact metric manifold is cosymplectic if and only if $\bar{\nabla} \phi$ vanishes identically, i.e., $\left(\bar{\nabla}_{X} \phi\right) Y=0$ and $\bar{\nabla}_{X} \xi=0$.

Proposition 2.1 [6] On a nearly cosymplectic manifold, the vector field $\xi$ is Killing.

From the above proposition, we have $g\left(\bar{\nabla}_{X} \xi, X\right)=0$ for any vector field $X$ tangent to $\bar{M}$, where $\bar{M}$ is a nearly cosymplectic manifold.

Let $M$ be a submanifold of an almost contact metric manifold $\bar{M}$ with induced metric $g$, and let $\nabla$ and $\nabla^{\perp}$ be the induced connections on the tangent bundle $T M$ and the normal bundle $T^{\perp} M$ of $M$, respectively. Denote by $\mathcal{F}(M)$ the algebra of smooth functions on $M$ and by $\Gamma(T M)$ the $\mathcal{F}(M)$-module of smooth sections of $T M$ over $M$. Then the Gauss and Weingarten formulas are given by

$$
\begin{aligned}
& \bar{\nabla}_{X} Y=\nabla_{X} Y+h(X, Y), \\
& \bar{\nabla}_{X} N=-A_{N} X+\nabla_{X}^{\perp} N,
\end{aligned}
$$


for each $X, Y \in \Gamma(T M)$ and $N \in \Gamma\left(T^{\perp} M\right)$, where $h$ and $A_{N}$ are the second fundamental form and the shape operator (corresponding to the normal vector field $N$ ), respectively, for the immersion of $M$ into $\bar{M}$. They are related as

$$
g(h(X, Y), N)=g\left(A_{N} X, Y\right),
$$

where $g$ denotes the Riemannian metric on $\bar{M}$ as well as induced on $M$.

For any $X \in \Gamma(T M)$, we write

$$
\phi X=T X+F X,
$$

where $T X$ is the tangential component and $F X$ is the normal component of $\phi X$.

A submanifold $M$ tangent to the structure vector field $\xi$ is said to be invariant (resp. anti-invariant) if $\phi\left(T_{x} M\right) \subset T_{x} M, \forall x \in M$ (resp. $\left.\phi\left(T_{x} M\right) \subset T_{x}^{\perp} M, \forall x \in M\right)$.

A submanifold $M$ tangent to the structure vector field $\xi$ of an almost contact metric manifold $\bar{M}$ is called a contact CR-submanifold (or semi-invariant submanifold) if there exists a pair of orthogonal differentiable distributions $\mathcal{D}$ and $\mathcal{D}^{\perp}$ on $M$ such that

(i) $T M=\mathcal{D} \oplus \mathcal{D}^{\perp} \oplus\langle\xi\rangle$, where $\langle\xi\rangle$ is the one-dimensional distribution spanned by $\xi$;

(ii) $\mathcal{D}$ is invariant under $\phi$, i.e., $\phi\left(\mathcal{D}_{x}\right) \subseteq \mathcal{D}_{x}, \forall x \in M$;

(iii) $\mathcal{D}^{\perp}$ is anti-invariant under $\phi$, i.e., $\phi\left(\mathcal{D}_{x}^{\perp}\right) \subset T_{x}^{\perp} M, \forall x \in M$.

A contact CR-submanifold is invariant if $\mathcal{D}^{\perp}=\{0\}$ and anti-invariant if $\mathcal{D}=\{0\}$, respectively. It is called a proper contact CR-submanifold if neither $\mathcal{D}=\{0\}$ nor $\mathcal{D}^{\perp}=\{0\}$. Moreover, if $\mu$ is the $\phi$-invariant subspace in the normal bundle $T^{\perp} M$, then in the case of a contact CR-submanifold, the normal bundle $T^{\perp} M$ can be decomposed as

$$
T^{\perp} M=F \mathcal{D}^{\perp} \oplus \mu
$$

Bishop and O'Neill [7] introduced the notion of warped product manifolds. They defined these manifolds as follows. Let $\left(N_{1}, g_{1}\right)$ and $\left(N_{2}, g_{2}\right)$ be two Riemannian manifolds and $f>0$ be a differentiable function on $N_{1}$. Consider the product manifold $N_{1} \times N_{2}$ with its projections $\pi_{1}: N_{1} \times N_{2} \rightarrow N_{1}$ and $\pi_{2}: N_{1} \times N_{2} \rightarrow N_{2}$. Then the warped product of $N_{1}$ and $N_{2}$ denoted by $M=N_{1} \times{ }_{f} N_{2}$ is a Riemannian manifold $N_{1} \times N_{2}$ equipped with the Riemannian structure such that

$$
g(X, Y)=g_{1}\left(\pi_{1 \star} X, \pi_{1 \star} Y\right)+\left(f \circ \pi_{1}\right)^{2} g_{2}\left(\pi_{2 \star} X, \pi_{2 \star} Y\right)
$$

for each $X, Y \in \Gamma(T M)$ and $\star$ is the symbol for the tangent map. Thus, we have

$$
g=g_{1}+f^{2} g_{2}
$$

The function $f$ is called the warping function of the warped product [7]. A warped product $N_{1} \times{ }_{f} N_{2}$ is said to be trivial if the warping function $f$ is constant.

We recall the following general result on warped product manifolds for later use.

Lemma 2.1 Let $M=N_{1} \times{ }_{f} N_{2}$ be a warped product manifold with the warping function $f$, then 
(i) $\nabla_{X} Y \in \Gamma\left(T N_{1}\right)$ is the lift of $\nabla_{X} Y$ on $N_{1}$,

(ii) $\nabla_{X} Z=\nabla_{Z} X=(X \ln f) Z$,

(iii) $\nabla_{Z} W=\nabla_{Z}^{N_{2}} W-g(Z, W) \nabla \ln f$

for each $X, Y \in \Gamma\left(T N_{1}\right)$ and $Z, W \in \Gamma\left(T N_{2}\right)$, where $\nabla \ln f$ is the gradient of the function $\ln f$ and $\nabla$ and $\nabla^{N_{2}}$ denote the Levi-Civita connections on $M$ and $N_{2}$, respectively.

\section{Contact CR-warped product submanifolds}

In this section, we consider the warped product submanifolds $M=N_{1} \times{ }_{f} N_{2}$ of a nearly cosymplectic manifold $\bar{M}$, where $N_{1}$ and $N_{2}$ are Riemannian submanifolds of $\bar{M}$. In the above product, if we assume $N_{1}=N_{T}$ and $N_{2}=N_{\perp}$, then the warped product of $N_{1}$ and $N_{2}$ becomes a contact CR-warped product. In this section, we discuss the contact CR-warped products and obtain an inequality for the squared norm of the second fundamental form. For the general case of warped product submanifolds of a nearly cosymplectic manifold, we have the following result.

Theorem 3.1 [8] A warped product submanifold $M=N_{1} \times{ }_{f} N_{2}$ of a nearly cosymplectic manifold $\bar{M}$ is a usual Riemannian product if the structure vector field $\xi$ is tangent to $N_{2}$, where $N_{1}$ and $N_{2}$ are the Riemannian submanifolds of $\bar{M}$.

If we consider $\xi \in \Gamma\left(T N_{1}\right)$, then for any $X \in \Gamma\left(T N_{2}\right)$, we have

$$
\bar{\nabla}_{X} \xi=\nabla_{X} \xi+h(X, \xi)
$$

Taking the inner product with $X \in \Gamma\left(T N_{2}\right)$, then by Proposition 2.1 and Lemma 2.1(ii), we obtain that $(\xi \ln f)\|X\|^{2}=0$. This means that either $\operatorname{dim} N_{2}=0$, which is not possible for warped products, or

$$
\xi \ln f=0 .
$$

Now, we consider the warped product contact CR-submanifolds of the types $M=N_{\perp} \times$ ${ }_{f} N_{T}$ and $M=N_{T} \times{ }_{f} N_{\perp}$ of a nearly cosymplectic manifold $\bar{M}$. In [8], the present author has proved that the warped product contact CR-submanifolds of the first type are usual Riemannian products of $N_{\perp}$ and $N_{T}$, where $N_{\perp}$ and $N_{T}$ are anti-invariant and invariant submanifolds of $\bar{M}$, respectively. In the following, we consider the contact CR-warped product submanifolds $M=N_{T} \times{ }_{f} N_{\perp}$ and obtain a general inequality. First, we have the following preparatory result for later use.

Lemma 3.1 [8] Let $M=N_{T} \times{ }_{f} N_{\perp}$ be a contact CR-warped product submanifold of a nearly cosymplectic manifold $\bar{M}$. If $X, Y \in \Gamma\left(T N_{T}\right)$ and $Z, W \in \Gamma\left(T N_{\perp}\right)$, then

(i) $g(h(X, Y), F Z)=0$,

(ii) $g(h(\phi X, Z), F Z)=(X \ln f)\|Z\|^{2}$.

If we replace $X$ by $\phi X$ in (ii) of Lemma 3.1, then we get

$$
g(h(X, Z), F Z)=-(\phi X \ln f)\|Z\|^{2} .
$$

For a Riemannian manifold of dimension $m$ and a smooth function $f$ on $M$, we recall 
(i) $\nabla f$, the gradient of $f$, is defined by

$$
g(\nabla f, X)=X(f), \quad \forall X \in \Gamma(T M) .
$$

(ii) $\Delta f$, the Laplacian of $f$, is defined by

$$
\Delta f=\sum_{i=1}^{m}\left\{\left(\nabla_{e_{i}} e_{i}\right) f-e_{i} e_{i}(f)\right\}=-\operatorname{div} \nabla f
$$

where $\nabla$ is the Levi-Civita connection on $M$ and $\left\{e_{1}, \ldots, e_{m}\right\}$ is an orthonormal frame on $M$.

As a consequence, we have

$$
\|\nabla f\|^{2}=\sum_{i=1}^{m}\left(e_{i}(f)\right)^{2}
$$

Now, we prove the main result of this section using the above results.

Theorem 3.2 Let $M=N_{T} \times{ }_{f} N_{\perp}$ be a contact CR-warped product submanifold of a nearly cosymplectic manifold $\bar{M}$. Then we have

(i) The length of the second fundamental form of $M$ satisfies the inequality

$$
\|h\|^{2} \geq 2 q\|\nabla \ln f\|^{2}
$$

where $q$ is the dimension of $N_{\perp}$ and $\nabla \ln f$ is the gradient of $\ln f$.

(ii) If the equality sign of (3.6) holds identically, then $N_{T}$ is a totally geodesic submanifold and $N_{\perp}$ is a totally umbilical submanifold of $\bar{M}$. Moreover, $M$ is a minimal submanifold of $\bar{M}$.

Proof Let $\bar{M}$ be a $(2 m+1)$-dimensional nearly cosymplectic manifold and $M=N_{T} \times{ }_{f} N_{\perp}$ be an $n$-dimensional contact CR-warped product submanifolds of $\bar{M}$. Let us consider the $\operatorname{dim} N_{T}=2 p+1$ and $\operatorname{dim} N_{\perp}=q$, then $n=2 p+1+q$. Let $\left\{e_{1}, \ldots, e_{p}, \phi e_{1}=e_{p+1}, \ldots, \phi e_{p}=\right.$ $\left.e_{2 p}, e_{2 p+1}=\xi\right\}$ and $\left\{e_{(2 p+1)+1}, \ldots, e_{n}\right\}$ be the local orthonormal frames on $N_{T}$ and $N_{\perp}$, respectively. Then the orthonormal frames in the normal bundle $T^{\perp} M$ of $F \mathcal{D}^{\perp}$ and $\mu$ are $\left\{F e_{(2 p+1)+1}, \ldots, F e_{n}\right\}$ and $\left\{e_{n+q+1}, \ldots, e_{2 m+1}\right\}$, respectively. Then the length of the second fundamental form $h$ is defined as

$$
\|h\|^{2}=\sum_{r=n+1}^{2 m+1} \sum_{i, j=1}^{n} g\left(h\left(e_{i}, e_{j}\right), e_{r}\right)^{2}
$$

For the assumed frames, the above equation can be written as

$$
\|h\|^{2}=\sum_{r=n+1}^{n+q} \sum_{i, j=1}^{n} g\left(h\left(e_{i}, e_{j}\right), e_{r}\right)^{2}+\sum_{r=n+q+1}^{2 m+1} \sum_{i, j=1}^{n} g\left(h\left(e_{i}, e_{j}\right), e_{r}\right)^{2} .
$$


The first term on the right-hand side of the above equality is the $F \mathcal{D}^{\perp}$-component and the second term is the $\mu$-component. Here, we equate the $F \mathcal{D}^{\perp}$-component, then we have

$$
\|h\|^{2} \geq \sum_{r=n+1}^{n+q} \sum_{i, j=1}^{n} g\left(h\left(e_{i}, e_{j}\right), e_{r}\right)^{2}
$$

The above equation can be written for the given frame of $F \mathcal{D}^{\perp}$ as

$$
\|h\|^{2} \geq \sum_{l=(2 p+1)+1}^{n} \sum_{i, j=1}^{n} g\left(h\left(e_{i}, e_{j}\right), F e_{l}\right)^{2}
$$

Let us decompose the above equation in terms of the components of $h(\mathcal{D}, \mathcal{D}), h\left(\mathcal{D}, \mathcal{D}^{\perp}\right)$ and $h\left(\mathcal{D}^{\perp}, \mathcal{D}^{\perp}\right)$, then we have

$$
\begin{aligned}
\|h\|^{2} \geq & \sum_{l=2 p+2}^{n} \sum_{i, j=1}^{2 p+1} g\left(h\left(e_{i}, e_{j}\right), F e_{l}\right)^{2} \\
& +2 \sum_{l=2 p+2}^{n} \sum_{i=1}^{2 p+1} \sum_{j=2 p+2}^{n} g\left(h\left(e_{i}, e_{j}\right), F e_{l}\right)^{2} \\
& +\sum_{l=2 p+2}^{n} \sum_{i, j=2 p+2}^{n} g\left(h\left(e_{i}, e_{j}\right), F e_{l}\right)^{2} .
\end{aligned}
$$

Using Lemma 3.1(i), the first term of (3.10) is identically zero and we shall compute the next term and leave the third term

$$
\|h\|^{2} \geq 2 \sum_{l=2 p+2}^{n} \sum_{i=1}^{2 p+1} \sum_{j=2 p+2}^{n} g\left(h\left(e_{i}, e_{j}\right), F e_{l}\right)^{2} .
$$

As $j, l=2 p+2, \ldots, n$, then we can write the above equation for one summation, and using (3.2), we obtain

$$
\|h\|^{2} \geq 2 \sum_{i=1}^{2 p+1} \sum_{l=2 p+2}^{n}\left(-\phi e_{i} \ln f\right)^{2} g\left(e_{l}, e_{l}\right)^{4} .
$$

Using the fact that $\xi$ is tangent to $N_{T}$ and $\xi \ln f=0$, the above equation can be written for the given frame of the distribution $\mathcal{D}$ as

$$
\|h\|^{2} \geq 2 \sum_{l=2 p+2}^{n}\left[\sum_{i=1}^{p}\left(e_{i} \ln f\right)^{2} g\left(e_{l}, e_{l}\right)^{4}+\sum_{i=1}^{p}\left(\phi e_{i} \ln f\right)^{2} g\left(e_{l}, e_{l}\right)^{4}\right] .
$$

Then from (3.5), the above expression will be

$$
\|h\|^{2} \geq 2 \sum_{l=2 p+2}^{n}\|\nabla \ln f\|^{2} g\left(e_{l}, e_{l}\right)^{4}=2 q\|\nabla \ln f\|^{2},
$$


which proves the inequality (3.6). Let us denote by $h^{\perp}$, the second fundamental form of $N_{\perp}$ in $M$, then by (2.4), we have

$$
g\left(h^{\perp}(Z, W), X\right)=g\left(\nabla_{Z} W, X\right)=-(X \ln f) g(Z, W)
$$

for any $X \in \Gamma\left(T N_{T}\right)$ and $Z, W \in \Gamma\left(T N_{\perp}\right)$. Thus, on using (3.3), we obtain

$$
g\left(h^{\perp}(Z, W), X\right)=g\left(\nabla_{Z} W, X\right)=-g(\nabla \ln f, X) g(Z, W),
$$

or equivalently,

$$
h^{\perp}(Z, W)=-g(Z, W) \nabla \ln f .
$$

Suppose the equality case holds in (3.6), then from (3.8) and (3.10), we obtain

$$
h(\mathcal{D}, \mathcal{D})=0, \quad h\left(\mathcal{D}^{\perp}, \mathcal{D}^{\perp}\right)=0, \quad h\left(\mathcal{D}, \mathcal{D}^{\perp}\right) \subset F \mathcal{D}^{\perp} .
$$

As $N_{T}$ is a totally geodesic submanifold in $M$ (by Lemma 2.1(i)), using this fact with the first part of (3.14), we get $N_{T}$ is totally geodesic in $\bar{M}$. On the other hand, the second condition of (3.14) with (3.13) implies that $N_{\perp}$ is totally umbilical in $\bar{M}$. Moreover, from (3.14), we get $M$ is a minimal submanifold of $\bar{M}$. This proves the theorem completely.

\section{Competing interests}

The authors declare that they have no competing interests.

\section{Authors' contributions}

SU carried out the whole research and drafted the manuscript. KAK has given the idea of this problem and checked the calculations. All authors read and approved the final manuscript.

\section{Author details}

${ }^{1}$ Institute of Mathematical Sciences, Faculty of Science, University of Malaya, Kuala Lumpur, 50603, Malaysia. ${ }^{2}$ School of Engineering \& Logistics, Faculty of Technology, Charles Darwin University, Darwin, NT 0909, Australia.

\section{Received: 3 July 2012 Accepted: 26 November 2012 Published: 18 December 2012}

\section{References}

1. Blair, DE: Almost contact manifolds with Killing structure tensors I. Pac. J. Math. 39, 285-292 (1971)

2. Blair, DE, Yano, K: Affine almost contact manifolds and $f$-manifolds with affine Killing structure tensors. Kodai Math. Semin. Rep. 23, 473-479 (1971)

3. Bonanzinga, V, Matsumoto, K: Warped product CR-submanifolds in locally conformal Kaehler manifolds. Period. Math. Hung. 48, 207-221 (2004)

4. Chen, BY: Geometry of warped product CR-submanifolds in Kaehler manifold. Monatshefte Math. 133, 177-195 (2001)

5. Mihai, I: Contact CR-warped product submanifolds in Sasakian space forms. Geom. Dedic. 109, 165-173 (2004)

6. Blair, DE, Showers, DK: Almost contact manifolds with Killing structure tensors II. J. Differ. Geom. 9, 577-582 (1974)

7. Bishop, RL, O'Neill, B: Manifolds of negative curvature. Trans. Am. Math. Soc. 145, 1-49 (1969)

8. Uddin, S, Kon, SH, Khan, MA, Singh, K: Warped product semi-invariant submanifolds of nearly cosymplectic manifolds. Math. Probl. Eng. 2011, 230374 (2011)

doi:10.1186/1029-242X-2012-304

Cite this article as: Uddin and Khan: An inequality for contact CR-warped product submanifolds of nearly cosymplectic manifolds. Journal of Inequalities and Applications 2012 2012:304. 